\title{
Increased Plasmodium falciparum Gametocyte Production in Mixed Infections with P. malariae
}

\author{
J. Teun Bousema,* Chris J. Drakeley, Petra F. Mens, Theo Arens, Rein Houben, Sabah A. Omar, Louis C. Gouagna, \\ Henk Schallig, and Robert W. Sauerwein \\ Department of Medical Microbiology, Radboud University, Nijmegen Medical Centre, Nijmegen, The Netherlands; Department of \\ Infectious and Tropical Diseases, London School of Hygiene and Tropical Medicine, London, United Kingdom; KIT Biomedical \\ Research, Royal Tropical Institute, Amsterdam, The Netherlands; Kenya Medical Research Institute, Nairobi, Kenya; Human Health \\ Division, International Centre of Insect Physiology and Ecology, Nairobi, Kenya; Unité de Recherche 016, Institut de Recherche pour \\ le Développement, Ouagadougou, Burkina Faso
}

\begin{abstract}
Plasmodium falciparum and $P$. malariae occur endemically in many parts of Africa. Observations from malariotherapy patients suggest that co-infection with $P$. malariae may increase $P$. falciparum gametocyte production. We determined $P$. falciparum gametocyte prevalence and density by quantitative nucleic acid sequence-based amplification (QT-NASBA) after antimalarial treatment of Kenyan children with either P. falciparum mono-infection or $P$. falciparum and $P$. malariae mixed infection. In addition, we analyzed the relationship between mixed species infections and microscopic $P$. falciparum gametocyte prevalence in three datasets from previously published studies. In Kenyan children, QT-NASBA gametocyte density was increased in mixed species infections $(P=0.03)$. We also observed higher microscopic prevalences of $P$. falciparum gametocytes in mixed species infections in studies from Tanzania and Kenya (odds ratio $=2.15,95 \%$ confidence interval $=0.99-4.65$ and $2.39,1.58-3.63$ ) but not in a study from Nigeria. These data suggest that co-infection with $P$. malariae is correlated with increased $P$. falciparum gametocytemia.
\end{abstract}

\section{INTRODUCTION}

Plasmodium falciparum and $P$. malariae are malaria species that occur endemically in many parts of sub Saharan Africa. Mixed infections with both species are usually observed in a small proportion of persons in cross-sectional studies $(2-$ $14 \%),{ }^{1-4}$ although their cumulative prevalence has been documented at $20-50 \%$ in longitudinal studies conducted over 1-2 years. $^{2-4}$ The co-occurrence of $P$. falciparum and $P$. malariae is often higher than would be expected on the basis of their individual parasite prevalence, ${ }^{5-8}$ and one parasite species may influence the infection dynamics of the other. The biologic and subsequent clinical interactions are therefore of interest, especially in light of vaccination trials specifically targeted at $P$. falciparum.

In mixed infections, the presence of $P$. malariae parasites can influence the disease manifestation of $P$. falciparum with a reduction in disease severity ${ }^{9}$ and a lower peak parasitemia, ${ }^{10}$ possibly as a consequence of heterologous immunity. ${ }^{11,12}$ Observations from malariotherapy patients indicate that co-infection with $P$. malariae may increase $P$. falciparum gametocytemia. ${ }^{13}$ If this increased gametocyte production is manifested in co-infections in field settings, this could have implications for the spread of malaria and malaria control. Predictably few field studies have addressed this issue: most clinical studies focus on $P$. falciparum mono-infections and use co-infection with other malaria species as an exclusion criterion. A study of 5,682 symptomatic patients in Thailand found that $P$. falciparum gametocytes were less common in mixed infections with $P$. vivax. ${ }^{14}$ A small cross-sectional study in 25 households in Mozambique suggested that $P$. falciparum gametocytes may also be less common in the presence of $P$. malariae. ${ }^{1}$ We examined the relation between $P$. falciparum gametocytemia and mixed infection with $P$. malariae in four

* Address correspondence to J. Teun Bousema, Department of Medical Microbiology 268, Radboud University Nijmegen Medical Centre, PO Box 9101, 6500 HB Nijmegen, The Netherlands. E-mail: t.bousema@ncmls.ru.nl separate studies. We compare $P$. falciparum gametocyte prevalence and density assessed by molecular methods after anti-malarial treatment of Kenyan children naturally infected with either $P$. falciparum mono-infection or $P$. falciparum and $P$. malariae mixed infection. In addition, we present analyses of the relationship between mixed species infections and microscopic $P$. falciparum gametocyte prevalence in three large datasets from previously published studies conducted in Tanzania, Kenya, and Nigeria.

\section{MATERIALS AND METHODS}

Gametocytemia and mixed species infections after antimalarial drug treatment. The study assessing $P$. falciparum gametocyte prevalence and density after antimalarial treatment was conducted from October to December 2004 in Mbita, a rural village on the shores of Lake Victoria in the Suba District of western Kenya. Transmission intensity is high and perennial in the study area (entomologic inoculation rate approximately six infectious bites per person per month $)^{15}$ with $P$. falciparum as predominant parasite species accounting for more than $95 \%$ of the clinical malaria cases. ${ }^{16}$ Patients with $P$. falciparum mono-infection were enrolled as part of a larger drug sensitivity study that was reported previously (Clinical Trials registration no. ISRCTN31291803 available from http:// www.controlled-trials.com/ISRCTN31291803). ${ }^{17}$ Children 6 months to 10 years of age with a temperature $>37.5 \mathrm{C}^{\circ}$ measured by earthermometer or a history of fever within the last 48 hours and with $P$. falciparum mono-infection at a density between 500 and 100,000 parasites/ $\mu \mathrm{L}$ were eligible for recruitment. Exclusion criteria were inability to take drugs orally, known hypersensitivity to any of the drugs given, reported treatment with antimalarial chemotherapy in the past two weeks, evidence of chronic disease or acute infection other than malaria, and domicile outside the study area and signs of severe malaria. Children with $P$. falciparum and $P$. malariae mixed infection were enrolled in the study on the basis of the same criteria but no minimum parasite density was used as an inclusion criterion. All subjects included in the 
analyses were treated with sulfadoxine-pyrimethamine (SP; Fansidar ${ }^{\circledR}$; Hoffmann LaRoche, Basel, Switzerland) plus amodiaquine (AQ), $10 \mathrm{mg} / \mathrm{kg}$, once a day for three days (Camoquine $^{\circledR}$; Pfizer, Dakar, Senegal) and subsequently followed for 28 days. The study protocol (SSC no. 791) was reviewed and approved by the Scientific Steering Committee and Ethical Review Committee of the Kenya Medical Research Institute, Nairobi, Kenya.

Microscopy and molecular parasite detection. Giemsastained blood smears were screened for asexual parasites and gametocytes at enrollment and on days 3, 7, 14, and 28 after treatment. Slides were declared negative if no parasites were observed in 100 microscopic fields, each containing approximately 15-20 leukocytes; asexual parasites and gametocytes were counted against 200 and 500 leukocytes, respectively. Conversion to parasites/microliter was made using a conversion rate of 8,000 leukocytes/ $\mu \mathrm{L}$. Parasite detection by quantitative nucleic acid sequence-based amplification (QTNASBA) was done for a random selection of $P$. falciparum mono-infections with complete follow-up (47 of 127) ${ }^{17}$ and for all $P$. falciparum plus $P$. malariae mixed infections ( 21 of 21). Nucleic acids were extracted from $50-\mu \mathrm{L}$ finger prick blood samples by the method described by Boom and others. ${ }^{18}$ Plasmodium falciparum QT-NASBA was performed on a NucliSens EasyQ analyzer (bioMérieux, Boxtel, The Netherlands) as described elsewhere for $P f_{s} 25$ mRNA. ${ }^{19} P f s 25$ mRNA is only expressed in stage V $P$. falciparum gametocytes $^{20}{ }^{20}$ and the $P f s 25$ QT-NASBA has a detection limit of 20-100 gametocytes/mL. Nuclisens Basic kits (bioMérieux) were used for amplification according to the manufacturer's instructions at a $\mathrm{KCl}$ concentration of $80 \mathrm{mM}$. A standard dilution series of in vitro cultured mature NF54 gametocytes ${ }^{21}$ was included in each run to ascertain gametocyte density. The presence of $P$. malariae parasites in mixed infections was confirmed by detecting $P$. malariae-specific $18 \mathrm{~S}$ ribosomal RNA using QT-NASBA. ${ }^{22}$

Data analyses. Nonparametric Wilcoxon rank sum tests were used to test differences between groups for statistical significance in case of continuous variables; chi-square tests were used for dichotomous variables. Multiple logistic regression models with generalized estimating equations (GEEs) were used to test the influence of $P$. falciparum plus $P$. $m a$ lariae mixed infection on $P f s 25$ QT-NASBA gametocyte prevalence. A similar procedure was carried out using GEEs for $P f s 25$ QT-NASBA gametocyte density. Estimates were adjusted for potential confounding factors (i.e., age, treatment outcome, microscopic asexual parasite density at enrollment, and fever at enrollment) and a random effect was included in the models to show correlations within persons. To quantify the influence of co-infection with $P$. malariae on $P$. falciparum gametocyte densities during follow-up, we determined the area under the curve (AUC) of $P f s 25$ QT-NASBA gametocyte density versus time. ${ }^{23,24}$ This measure incorporates both the magnitude and the duration of transmission potential and was described by Mendez and others. ${ }^{24}$ The AUC from days 0 to 42 was calculated as AUC $=[(3-0) \times$ $\left(\mathrm{g}_{0}+\mathrm{g}_{3}\right) / 2+(7-3) \times\left(\mathrm{g}_{3}+\mathrm{g}_{7}\right) / 2+(14-7) \times\left(\mathrm{g}_{7}+\mathrm{g}_{14}\right) / 2+$ $\left.(28-14) \times\left(g_{14}+g_{28}\right) / 2\right] / 28$ where $g_{d}$ represents $P f s 25$ QTNASBA gametocyte density on day d. Gametocyte negative samples were included as zeroes. The measure was scaled by 28 so that it represents AUC per day and this was transformed by $\log _{10}$ for comparisons. Statistical analyses were performed using procedures available in SPSS version 12.0 (SPSS Inc., Chicago, IL) and Stata version 8.0 (Stata Corporation, College Station, TX).

Analyses on data from three previously conducted studies. Data from three studies were analyzed on the basis of the availability of information on the prevalence of $P$. falciparum asexual parasites and gametocytes and $P$. malariae asexual parasites.

The first dataset was obtained from a series of crosssectional studies in six altitude transects (150-1,800 meters) in the Kilimanjaro and Tanga regions in northern Tanzania. ${ }^{25}$ Cross-sectional surveys in individuals 6 months to 45 years of age were conducted twice in a period of 6 months; $100 \mathrm{mi}-$ croscopic fields were screened for asexual parasites of $P$. falciparum and $P$. malariae and gametocytes of $P$. falciparum. Asexual and gametocyte densities were recorded per 200 leukocytes and 500 leukocytes, respectively.

The second dataset was obtained from a longitudinal study in Mbita in western Kenya. ${ }^{26}$ Children 6 months to 16 years of age were screened weekly for five weeks; 100 microscopic fields were screened for asexual parasites of $P$. falciparum and $P$. malariae and gametocytes of $P$. falciparum.

The third dataset was obtained from the Garki dataset from 1970 and 1971, the years prior to the transmission-reducing intervention (http://www.sti.ch/research/biostatistics/ downloads.html). ${ }^{27}$ In this part of the study, surveys were conducted in the general population (0-72 years of age) of villages in Garki in northern Nigeria. Every ten weeks slides were collected and 200 or 400 microscopic fields were screened for asexual parasites of $P$. falciparum and $P$. $m a-$ lariae and gametocytes of $P$. falciparum. Parasite densities were calculated as the percentage of positive fields. Sampling took place every 10 weeks.

Microscopic gametocyte density data were available for the Kenyan and Tanzanian datasets but because there was little variation in these densities (typically 16-32 gametocytes/ $\mu \mathrm{L}$ ), we considered it appropriate to present microscopic gametocyte prevalence data only. Although the sampling interval in the Tanzanian and Nigerian datasets was relatively large, this may not guarantee independence of observations. ${ }^{28}$ Therefore, multiple logistic regression models with GEEs were initially used to test the relation between $P$. falciparum gametocyte prevalence and the presence of $P$. falciparum and $P$. malariae mixed infections in all data sets. Estimates were adjusted for potential confounding factors and a random effect was included in the models to show correlations within persons. If the addition of the random effect did not improve the model, as was the case for data from Tanzania, outcomes of conventional logistic regression models were presented.

\section{RESULTS}

Gametocytemia and mixed species infections after antimalarial drug treatment. In Mbita, Kenya, 127 P. falciparum mono-infections and $21 P$. falciparum and $P$. malariae mixed infections were treated with SP plus AQ. The presence of $P$. malariae parasites was confirmed by $P$. malariae $18 \mathrm{~S}$ ribosomal RNA QT-NASBA in all mixed species infections (21 of 21 ) and in none of the $P$. falciparum mono-infections ( 0 of 47) tested. Complete 28-day follow-up data were available for 115 $P$. falciparum mono-infections and 20 mixed species infec- 
tions. An adequate clinical response was observed in $87.0 \%$ (100 of 115) of the $P$. falciparum mono-infections and $100 \%$ (20 of 20) of the mixed species infections $(P=0.13)$. Children with mixed species infections were significantly older than those with $P$. falciparum mono-infections $(P=0.007$; Table 1). Microscopic $P$. falciparum gametocyte prevalence was non-significantly higher in mixed species infections $(P=$ 0.26; Table 1).

Gametocyte prevalence by $P f s 25$ QT-NASBA was $91.1 \%$ (41 of 45) in $P$. falciparum mono-infections at enrollment and $90.5 \%$ (19 of 21$)$ in mixed species infections $(P=0.93$; Table 1). The $P f s 25$ QT-NASBA gametocyte prevalence at enrollment was negatively associated with age $(\beta=-0.36, \mathrm{SE}=$ $0.17, P=0.04)$ and showed a weak positive association with microscopic asexual parasite density $(\beta=0.38, \mathrm{SE}=0.35$, $P=0.28)$. On the third day after the initiation of treatment, $P f$ s25 QT-NASBA gametocyte prevalence was non-significantly higher in mixed species infections $(P=0.07)$ (Figure 1A) without any clear difference during the rest of follow-up. When the entire period of follow-up was considered, there was no statistically significant difference in the prevalence of gametocytes by $P f s 25$ QT-NASBA between $P$. falciparum mono-infections and mixed species infections (GEE $\beta=0.21$, $\mathrm{SE}=0.42, P=0.62)$, after adjustment for age and asexual parasite density at enrollment. However, in $P f s 25$ QTNASBA gametocyte carriers, the $P f s 25$ QT-NASBA gametocyte density was consistently increased in mixed species infections (Figure 1B). The geometric mean Pfs25 QT-NASBA density was more than two-fold higher on the third day after initiation of treatment in mixed species infections: 2.9 gametocytes $/ \mu \mathrm{L}(95 \%$ confidence interval $[\mathrm{CI}]=0.7-12.4)$ for $P$. falciparum mono-infections and 8.2 gametocytes $/ \mu \mathrm{L}(95 \%$ $\mathrm{CI}=3.1-21.3)$ for mixed species infections $(P=0.11)$. The $P f s 25$ QT-NASBA gametocyte density throughout the study period was significantly higher in mixed infections than in mono-infections (GEE $\beta=0.38, \mathrm{SE}=0.17, P=0.03$ ) after adjustment for age and microscopic $P$. falciparum asexual parasite density at enrollment. Treatment outcome and the presence of fever at enrollment did not confound this relationship.

The transmission potential during follow-up, quantified as the AUC of $P f s 25$ QT-NASBA gametocyte density versus time, was significantly higher for mixed species infections $(P=0.02$; Table 2). The number of sampling times when gametocytes were detected by $P f s 25$ QT-NASBA was not significantly different between the two groups $(P=0.45)$, but
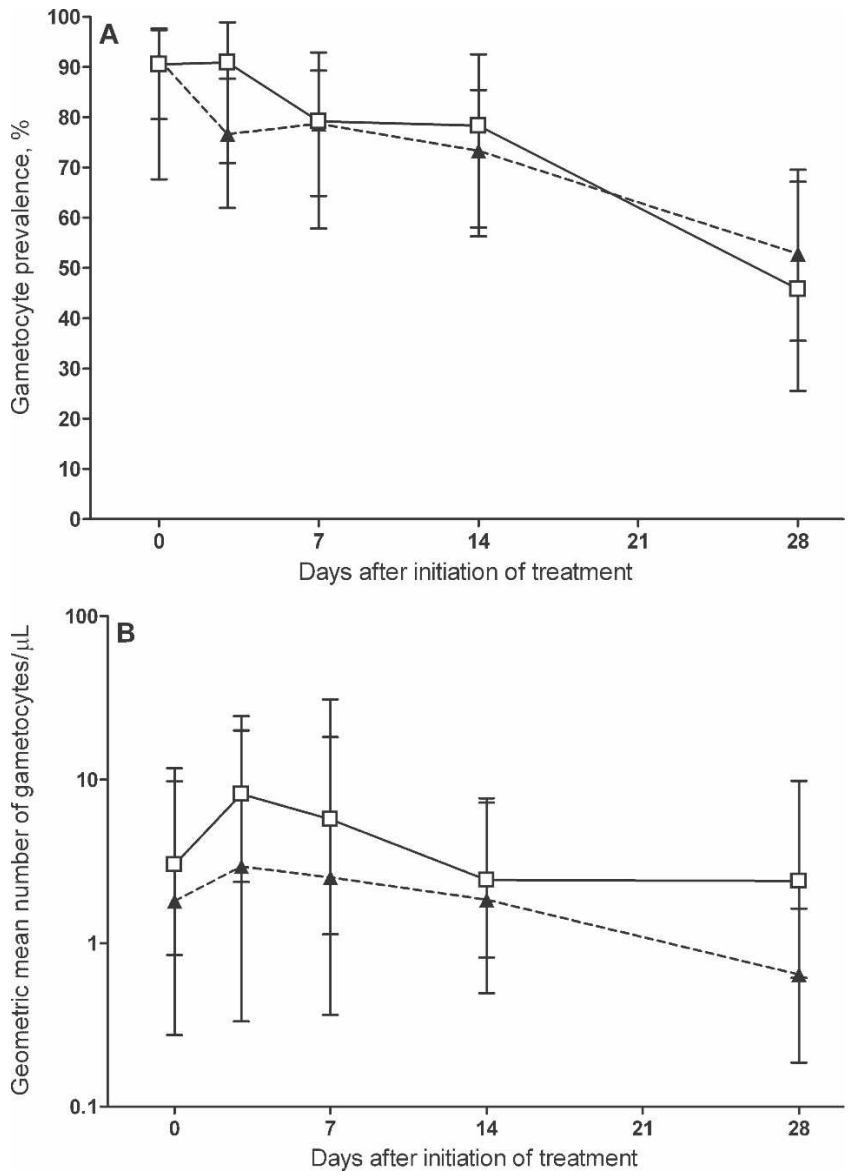

FIGURE 1. Plasmodium falciparum gametocyte prevalence and density determined by Pfs 25 quantitative nucleic acid sequence-based amplification (QT-NASBA) in $P$. falciparum mono-infections and mixed infections with $P$. malariae. Shown are QT-NASBA gametocyte prevalence (A) and density (B) for P. falciparum monoinfections (closed triangles, broken line) and $P$. falciparum plus $P$. malariae mixed infections (open squares, solid line). Bars indicate the $95 \%$ confidence intervals.

the geometric mean $P f s 25$ QT-NASBA gametocyte density in $P f_{s} 25$ QT-NASBA gametocyte-positive samples was higher in mixed species infections $(P=0.03$; Table 2$)$.

Gametocyte prevalence in mixed species infections in previously conducted studies. We analyzed three datasets on the relationship between $P$. falciparum and $P$. malariae mixed

TABLE 1

Baseline characteristics of children with Plasmodium falciparum mono-infection or $P$. falciparum and $P$. malariae mixed infection*

\begin{tabular}{|c|c|c|c|}
\hline Characteristic & $P$. falciparum mono-infection & P. falciparum and $P$. malariae mixed infection & $P$ \\
\hline No. of children & 127 & 21 & \\
\hline Age, years, median (IQR) & $2.6(1.4-4.9)$ & $4.4(2.3-6.0)$ & 0.007 \\
\hline Sex, \% male $(\mathrm{n} / \mathrm{N})$ & $52.8(67 / 127)$ & $52.4(11 / 21)$ & 0.98 \\
\hline Fever, \% (n/N) & $53.5(68 / 127)$ & $38.1(8 / 21)$ & 0.19 \\
\hline $\mathrm{Hb}$, median (IQR) & $9.3(8.0-10.7)$ & $10.4(8.5-11.0)$ & 0.11 \\
\hline \multicolumn{4}{|l|}{ P. malariae } \\
\hline Microscopic asexual parasite density, GM (IQR) & - & $1,419(800-2,600)$ & - \\
\hline \multicolumn{4}{|l|}{ P. falciparum } \\
\hline Microscopic asexual parasite density, GM (IQR) & $11,452(5,040-31,679)$ & $6,400(3,143-16,858)$ & 0.03 \\
\hline Microscopic gametocyte prevalence, \% (n/N) & $22.3(27 / 121)$ & $35.0(7 / 20)$ & 0.26 \\
\hline Pfs25 QT-NASBA gametocyte prevalence, \% (n/N) & $91.1 \%(41 / 45)$ & $90.5 \%(19 / 21)$ & 0.93 \\
\hline$P f s 25$ QT-NASBA gametocyte density, GM (IQR) & $1.8(0.3-9.7)$ & $3.0(0.8-11.7)$ & 0.44 \\
\hline
\end{tabular}


TABLE 2

$P f_{s} 25$ QT-NASBA gametocyte carriage during follow-up for children with Plasmodium falciparum mono-infection or $P$. falciparum and $P$. malariae mixed infection*

\begin{tabular}{|c|c|c|c|}
\hline Characteristic & P. falciparum mono-infection & P. falciparum and P. malariae mixed infection & $P$ \\
\hline Mean AUC of gametocyte density/ $\mu \mathrm{L}$ vs. time, (IQR) & $0.9(0.1-8.4)$ & $5.5(3.3-18.1)$ & $0.02 \dagger$ \\
\hline No. of sampling times when gametocytes were detected, $\%(\mathrm{n} / \mathrm{N})$ & $74.5(158 / 212)$ & $79.4(81 / 102)$ & $0.45 \dagger+$ \\
\hline $\begin{array}{l}\text { GM gametocyte density/ } / \mu \mathrm{L} \text { on days when gametocytes were } \\
\text { detected (IQR) }\end{array}$ & $1.9(0.4-13.0)$ & $4.0(1.0-14.3)$ & $0.03+t$ \\
\hline
\end{tabular}

* QT-NASBA = quantitative nucleic acid sequence-based amplification; AUC = area under the curve; IQR = interquartile range; GM = geometric mean

$\dagger$ Adjusted for log-transformed asexual parasite density at enrollment and age

\$ Adjusted for correlations between observations from the same person.

infection and the prevalence of $P$. falciparum gametocytes. Prevalence data were available from multiple time points. Data were presented for all time points combined; the fact that multiple observations were derived from the same persons was taken into account in the statistical analysis. There was considerable variation in the strength of the association between microscopic $P$. falciparum gametocyte prevalence and mixed species infection (Table 3 ). We observed a borderline significant increase in $P$. falciparum gametocyte prevalence for mixed species infections in data from different sites in north eastern Tanzania (odds ratio [OR] $=2.15,95 \%$ $\mathrm{CI}=0.99-4.65)$ and this increase was statistically significant for data from Kenya $(\mathrm{OR}=2.39,95 \% \mathrm{CI}=1.58-3.63)$, after adjustment for age and concurrent microscopic asexual parasite density. In the dataset from Nigeria, the effect varied between different age groups. There was a borderline significant lower microscopic $P$. falciparum gametocyte prevalence in mixed species infections for persons less than 10 years of age $(\mathrm{OR}=0.88,95 \%$ CI $0.56-1.00)$, and there was a weak and non-significant higher risk in older children after adjustment for age and concurrent microscopic $P$. falciparum asexual parasite density $(\mathrm{OR}=1.13,95 \%$ CI $0.87-1.48)$.

\section{DISCUSSION}

In this study, we show that co-infection with $P$. malariae is correlated with an increase in $P$. falciparum gametocytes. This finding is consistent in three different studies from different malaria-endemic areas, using both microscopy and molecular methods; the effect was not apparent in a fourth study.

In Kenyan children with symptomatic malaria, we observed a non-significantly higher microscopic $P$. falciparum gametocyte prevalence prior to treatment. Although Pfs25 QTNASBA gametocyte prevalence was not significantly increased, $P f s 25$ QT-NASBA gametocyte density was consistently higher in mixed species infections throughout one month of follow-up. This finding could not be explained by differences in treatment efficacy, age, fever, or enrollment parasite density. Kenyan children with mixed species infec- tions were more likely to respond well to treatment, were older, and had lower P. falciparum asexual parasite densities at enrollment; all factors previously associated with lower rather than higher gametocyte prevalence and density. ${ }^{26,29}$

In $P$. falciparum, most gametocytes that appear after treatment are likely to have been committed to sexual stage development prior to treatment. Gametocytes take 8-12 days to develop ${ }^{30}$ and the peak in gametocyte prevalence and density that is commonly seen after treatment is at least partly the result of an efflux of these sequestered gametocytes. ${ }^{31} \mathrm{Al}-$ though a more pronounced release of gametocytes in the presence of $P$. malariae co-infection could explain our findings after antimalarial treatment, it seems more plausible that mixed species infections have a higher commitment to production of $P$. falciparum gametocytes that persist after treatment, without a causative role for treatment as such. This finding is also suggested by the non-significantly increased microscopic gametocyte prevalence and $P f s 25$ QT-NASBA gametocyte density in mixed species infections prior to treatment.

Retrospective analysis of data from previously conducted epidemiologic studies show a similar picture. In studies conducted in Kenya and Tanzania, we observed a significantly higher microscopic prevalence of $P$. falciparum gametocytes in mixed species infections. Because microscopy underestimates the total proportion of gametocyte carriers and only detects relatively high gametocyte densities, ${ }^{19}$ these microscopic findings can be interpreted as a higher prevalence of high density gametocyte carriage in mixed species infections.

The Nigerian Garki study did not confirm the relationship between $P$. falciparum gametocytemia and mixed species infections. In contrast, there was a non-significantly lower $P$. falciparum gametocyte prevalence associated with mixed species infection in children less than 10 years of age, ${ }^{1}$ and there was a weak and non-significant increased risk of $P$. falciparum gametocyte carriage in older persons with mixed species infections. This finding indicates that the relationship between mixed species infections and gametocytemia may be different under different endemicities. Despite this consideration, our

TABLE 3

Re-examination of studies detecting Plasmodium falciparum gametocytes in mono-infections and $P$. falciparum plus $P$. malariae mixed infections*

\begin{tabular}{|c|c|c|c|c|}
\hline Characteristic & $\begin{array}{l}\text { Tanzania, 2005, all } \\
\text { age groups }\end{array}$ & $\begin{array}{l}\text { Kenya, 2001, all } \\
\text { age groups }\end{array}$ & $\begin{array}{c}\text { Nigeria, } 1970-1971, \\
<10 \text { years }\end{array}$ & $\begin{array}{l}\text { Nigeria, } 1970-1971 \\
\quad \geq 10 \text { years }\end{array}$ \\
\hline P. falciparum mono-infection, \% (n/N) & $9.3(187 / 2,007)$ & $28.4(342 / 1,206)$ & $34.7(1203 / 3,462)$ & $9.6(422 / 4,400)$ \\
\hline$P$. falciparum and $P$. malariae mixed infection, $\%(\mathrm{n} / \mathrm{N})$ & $17.0(9 / 53)$ & $58.6(68 / 116)$ & $32.1(604 / 1,880)$ & $13.1(81 / 619)$ \\
\hline OR $(95 \%$ CI $)$ & $2.15(0.99-4.65) \dagger$ & $2.39(1.58-3.63) \dagger$ & $0.88(0.56-1.00) \dagger+$ & $1.13(0.87-1.48) \dagger+$ \\
\hline$P$ value & $0.05 \dagger$ & $<0.001 \dagger+$ & $0.05 \dagger+$ & $0.37 \dagger \ddagger$ \\
\hline
\end{tabular}

$*$ OR $=$ odds ratio; $\mathrm{CI}=$ confidence interval.

$\dagger$ Adjusted for concurrent log-transformed asexual $P$. falciparum parasite density and age in years.

$\$$ Adjusted for correlations between observations from the same individual. Data were derived from previously published studies that were conducted in Tanzania, ${ }^{24}$ Kenya, ${ }^{25}$ and Nigeria. ${ }^{26}$ 
findings in three independent datasets strengthen our conclusion that $P$. falciparum gametocyte production may be higher in the presence of $P$. malariae parasites. This would confirm observations from malariotherapy patients where co-infection with $P$. malariae stimulates $P$. falciparum gametocyte production. ${ }^{13}$ A possible influence in the other direction, i.e., a change in $P$. malariae gametocytemia in mixed species infections, could not be determined. Plasmodium malariae gametocytes were not recorded in any of the studies discussed in this report and a molecular tool to detect sexual stage parasites of other species than $P$. falciparum is currently unavailable.

Although three different studies show a similar relationship between mixed species infections and $P$. falciparum gametocytemia, our findings should be interpreted with caution. Malaria-associated leukopenia may have impaired our estimate of microscopic gametocyte density in Kenyan children with symptomatic malaria, where a standard leukocyte concentration of 8,000 cells $/ \mu \mathrm{L}$ was assumed. ${ }^{32}$ Moreover, the extent of leukopenia may differ between different plasmodium spe$\operatorname{cies}^{32}$ and could therefore confound relationships between mixed species infections and microscopically estimated asexual parasite or gametocyte densities. Leukocyte counts are, however, unlikely to have influenced the major outcome measures of this study, which were $P f_{s} 25$ QT-NASBA gametocyte density and prevalence and microscopic gametocyte prevalence. In addition, none of the conducted studies were specifically designed to detect interactions between malaria species. This would ideally require longitudinal studies in the absence of antimalarial treatment. Our design hampers us to draw conclusions on causality in the observed relations. Plasmodium falciparum infections show parasitic waves that may be followed by an increased gametocyte production. ${ }^{33}$ Similarly, $P$. malariae parasite densities may increase when $P$. falciparum densities decrease. ${ }^{34}$ This may result in a simultaneous increase in concentrations of $P$. malariae asexual parasites and $P$. falciparum gametocytes, making them more likely to be detected by microscopy, without any causal relationship between the two.

If there is a causal relationship between mixed species infections and gametocyte production, it is likely to be mediated by cross-species immune responses. Mixed genotype infections have been associated with higher transmission success and higher gametocytemia in animal models. ${ }^{35}$ A similar phenomenon may play a role in mixed species infections and may indicate a response of parasites in terms of transmission potential in the presence of a competitor. Although antibodymediated parasite immunity seems to be largely species and strain-specific, it is likely that there is a certain degree of cross-reactive immunity between species. ${ }^{36,37}$ There is evidence for heterologous protective immunity for $P$. falciparum induced by $P$. malariae $e^{9,37}$ and for cross-species regulation of parasite densities. ${ }^{12,38,39}$ This cross-species immunity may also influence malaria transmission. ${ }^{36}$ A higher infectivity of parasites to mosquitoes in the presence of a different malaria species was observed for simian malaria species ${ }^{40}$ and for human $P$. vivax and P. falciparum in Aotus monkeys. ${ }^{41} \mathrm{Al}-$ though we did not determine immune responses, we hypothesize that cross-reactive antibodies may play a role in explaining our findings. Antibodies that are cross-reactive between species may increase the immune stress experienced by $P$. falciparum, thereby stimulating this species to invest in trans- mission stages. The observation from the Garki dataset that increased gametocyte prevalence is only observed in children more than 10 years of age may be related to the development of clinical immunity in this age group. Further analysis allowing for the effects of transmission intensity of both $P$. falciparum and $P$. malariae is warranted but beyond the scope of this report.

The relevance of our findings for malaria transmission depends on prevalence of mixed species infections and influence of an increase in gametocyte density on malaria transmission potential. We have recently shown a positive association between gametocyte density and the proportion of infected mosquitoes, including submicroscopic gametocyte densities. ${ }^{42}$ Thus, the higher densities of gametocytes (and the higher prevalence of high density gametocyte carriage) in mixed species infections are likely to result in a higher proportion of infected mosquitoes. The infectiousness of gametocytes in symptomatic Kenyan children was confirmed on day 14 after the initiation of treatment, with 23 of 28 children with $P$. falciparum mono-infections ${ }^{17}$ and 3 of 4 children with $P$. falciparum and $P$. malariae mixed infections infecting at least one mosquito. The prevalence of mixed species infections in a given population is highly variable. Plasmodium falciparum and $P$. malariae mixed infections may account for $6 \%$ of the $P$. falciparum malaria cases in western Kenya, ${ }^{43}$ but the prevalence may be much higher in longitudinal studies ${ }^{2-4}$ or in studies using species specific molecular detection techniques. ${ }^{1,44}$ In our study, we found no sub-patent $P$. malariae infections in children with apparent $P$. falciparum monoinfections although a study from Mozambique found a twofold increase in the proportion of mixed species infections using a polymerase chain reaction. ${ }^{1}$ The prevalence of $P$. $m a-$ lariae infections may further increase when $P$. falciparumspecific control programs are implemented, ${ }^{2}$ such as current vaccine trials.

In conclusion, our data suggest that the transmission potential of $P$. falciparum is increased by co-infection with $P$. malariae. Longitudinal studies are needed to confirm this relationship, to identify possible mechanisms, and to determine the duration and relevance of the potential increase in malaria transmission that we observe. These longitudinal studies should preferably use molecular tools to detect and quantify the different stages of parasite species in the absence of malaria treatment.

Received May 7, 2007. Accepted for publication November 19, 2007.

Acknowledgments: We thank the community of Mbita for their cooperation; and S. Kaniaru (Kenya Medical Research Institute), G. Omweri, N. Makio, P. Sawa, B. Kapesa, K. Okoth and P. Ongele (International Centre of Insect Physiology and Ecology) for their work at the clinic and in the field. We also thank the Joint Malaria Programme (a collaboration between the Tanzanian National Institute for Medical Research; Kilimanjaro Christian Medical Centre; the London School of Hygiene and Tropical Medicine, and the Centre for Medical Parasitology, University of Copenhagen) for access to the data from the Usambara Mountains.

Financial support: J. Teun Bousema is supported by The Netherlands Foundation for the Advancement of Tropical Research (W 07.05.203.00) through Poverty Related Infection Oriented Research, and Chris J. Drakeley is supported by a research fellowship in tropical medicine (\#063516) from the Wellcome Trust.

Authors' addresses: J. Teun Bousema, Theo Arens, and Robert W. Sauerwein, Department of Medical Microbiology 268, Radboud University Nijmegen Medical Centre, PO Box 9101, 6500 HB, The Neth- 
erlands, Telephone: 31-24-361-9515, E-mails: t.bousema@ncmls.ru.nl, t.arens@mmb.umcn.nl, and r.sauerwein@mmb.umcn.nl. Chris J. Drakeley and Rein Houben, Department of Infectious and Tropical Diseases, London School of Hygiene and Tropical Medicine, London WC1E 7HT, United Kingdom, E-mails: chris.drakeley@lshtm.ac.uk and rein.houben@1shtm.ac.uk. Petra F. Mens and Henk Schallig, KIT Biomedical Research, Royal Tropical Institute, Amsterdam, The Netherlands, E-mails: p.mens@kit.nl and h.schallig@kit.nl. Sabah A. Omar, Kenya Medical Research Institute, PO Box 54840, Nairobi, Kenya, E-mail: osabah@kemri.org. Louis C. Gouagna, Institut de Recherche pour le Développement, 01 PO Box 182, Ouagadougou, Burkina Faso, E-mail: louis-clement.gouagna@ird.bf.

\section{REFERENCES}

1. Marques PX, Saute F, Pinto VV, Cardoso S, Pinto J, Alonso PL, Rosario VE, Arez AP, 2005. Plasmodium species mixed infections in two areas of Manhica District, Mozambique. Int J Biol Sci 1: 96-102.

2. Bruce-Chwatt LJ, 1963. A longitudinal survey of natural malaria infection in a group of West African adults. West Afr Med J 12: 199-217.

3. Miller MJ, 1958. Observations on the natural history of malaria in the semi-resistant West African. Trans R Soc Trop Med Hyg 52: $152-168$.

4. Wilson DB, 1936. Rural hyper-endemic malaria in Tanganyika territory. Trans $R$ Soc Trop Med Hyg 6: 583-618.

5. Arez AP, Pinto J, Palsson K, Snounou G, Jaenson TG, do Rosario V, 2003. Transmission of mixed Plasmodium species and Plasmodium falciparum genotypes. Am J Trop Med Hyg 68: 161-168.

6. Molineaux L, Storey J, Cohen JE, Thomas A, 1980. A longitudinal study of human malaria in the West African Savanna in the absence of control measures: relationships between different Plasmodium species, in particular P. falciparum and P. malariae. Am J Trop Med Hyg 29: 725-737.

7. Barnish G, Maude GH, Bockarie MJ, Erunkulu OA, Dumbuya MS, Greenwood BM, 1993. Malaria in a rural area of Sierra Leone. II. Parasitological and related results from pre- and post-rains clinical surveys. Ann Trop Med Parasitol 87: 137148.

8. Pinto J, Sousa CA, Gil V, Goncalves L, Lopes D, do Rosario V, Charlwood JD, 2000. Mixed-species malaria infections in the human population of Sao Tome island, west Africa. Trans $R$ Soc Trop Med Hyg 94: 256-257.

9. Black J, Hommel M, Snounou G, Pinder M, 1994. Mixed infections with Plasmodium falciparum and $P$ malariae and fever in malaria. Lancet 343: 1095.

10. Mason DP, McKenzie FE, Bossert WH, 1999. The blood-stage dynamics of mixed Plasmodium malariae-Plasmodium falciparum infections. $J$ Theor Biol 198: 549-566.

11. Richie TL, 1998. Interactions between malaria parasites infecting the same vertebrate host. Parasitology 96: 607-639.

12. Bruce MC, Day KP, 2003. Cross-species regulation of Plasmodium parasitaemia in semi-immune children from Papua New Guinea. Trends Parasitol 19: 271-277.

13. McKenzie FE, Jeffery GM, Collins WE, 2002. Plasmodium malariae infection boosts Plasmodium falciparum gametocyte production. Am J Trop Med Hyg 67: 411-414.

14. Price R, Nosten F, Simpson JA, Luxemburger C, Phaipun L, ter Kuile F, van Vugt M, Chongsuphajaisiddhi T, White NJ, 1999. Risk factors for gametocyte carriage in uncomplicated falciparum malaria. Am J Trop Med Hyg 60: 1019-1023.

15. Mutero CM, Ouma JH, Agak BK, Wanderi JA, Copeland RS, 1998. Malaria prevalence and use of self-protection measures against mosquitoes in Suba District, Kenya. East Afr Med J 75: $11-15$.

16. Gouagna LC, Okech BA, Kabiru EW, Killeen GF, Obare P, Ombonya S, Beier JC, Knols BG, Githure JI, Yan G, 2003. Infectivity of Plasmodium falciparum gametocytes in patients attending rural health centres in western Kenya. East Afr Med $J$ 80: 627-634.

17. Bousema JT, Schneider P, Gouagna LC, Drakeley CJ, Tostmann A, Houben R, Githure JI, Ord R, Sutherland CJ, Omar SA,
Sauerwein RW, 2006. Moderate effect of artemisinin-based combination therapy on transmission of Plasmodium falciparum. J Infect Dis 193: 1151-1159.

18. Boom R, Sol CJ, Salimans MM, Jansen CL, Wertheim-van Dillen PM, van der Noordaa J, 1990. Rapid and simple method for purification of nucleic acids. J Clin Microbiol 28: 495-503.

19. Schneider P, Bousema T, Omar S, Gouagna L, Sawa P, Schallig H, Sauerwein R, 2006. (Sub)microscopic Plasmodium falciparum gametocytaemia in Kenyan children after treatment with sulphadoxine-pyrimethamine monotherapy or in combination with artesunate. Int J Parasitol 36: 403-408.

20. Babiker HA, Abdel-Wahab A, Ahmed S, Suleiman S, RanfordCartwright L, Carter R, Walliker D, 1999. Detection of low level Plasmodium falciparum gametocytes using reverse transcriptase polymerase chain reaction. Mol Biochem Parasitol 99: $143-148$.

21. Ponnudurai T, Lensen AH, Leeuwenberg AD, Meuwissen JH, 1982. Cultivation of fertile Plasmodium falciparum gametocytes in semi-automated systems. 1 . Static cultures. Trans $R$ Soc Trop Med Hyg 76: 812-818.

22. Mens PF, Schoone GJ, Kager PA, Schallig HD, 2006. Detection and identification of human Plasmodium species with realtime quantitative nucleic acid sequence-based amplification. Malar J 5: 80.

23. Dunyo S, Milligan P, Edwards T, Sutherland C, Targett G, Pinder M, 2006. Gametocytaemia after drug treatment of asymptomatic Plasmodium falciparum. PloS Clinical Trials 1: e20.

24. Mendez F, Munoz A, Plowe CV, 2006. Use of area under the curve to characterize transmission potential after antimalarial treatment. Am J Trop Med Hyg 75: 640-646.

25. Drakeley CJ, Carneiro I, Reyburn H, Malima R, Lusingu JP, Cox J, Theander TG, Nkya WM, Lemnge MM, Riley EM, 2005. Altitude-dependent and -independent variations in Plasmodium falciparum prevalence in northeastern Tanzania. $J$ Infect Dis 191: 1589-1598.

26. Bousema JT, Gouagna LC, Drakeley CJ, Meutstege AM, Okech BA, Akim IN, Beier JC, Githure JI, Sauerwein RW, 2004. Plasmodium falciparum gametocyte carriage in asymptomatic children in western Kenya. Malar J 3: 18.

27. Molineaux L, Gramiccia G, 1980. The Garki Project. Research on the Epidemiology and Control of Malaria in the Sudan Savannah of West Africa. Geneva: World Health Organization.

28. Cohen JE, Singer B, 1979. Malaria in Nigeria: constrained continuous-time Markov models for discrete-time longitudinal data on human mixed-species infections. Levin SA, ed. Some Mathematical Questions in Biology. Providence, RI: American Mathematical Society, 12: 69-133.

29. Akim NI, Drakeley C, Kingo T, Simon B, Senkoro K, Sauerwein $\mathrm{RW}, 2000$. Dynamics of $P$. falciparum gametocytemia in symptomatic patients in an area of intense perennial transmission in Tanzania. Am J Trop Med Hyg 63: 199-203.

30. Sinden RE, 1983. The cell biology of sexual development in plasmodium. Parasitology 86: 7-28.

31. Drakeley C, Sutherland C, Bousema JT, Sauerwein RW, Targett GA, 2006. The epidemiology of Plasmodium falciparum gametocytes: weapons of mass dispersion. Trends Parasitol 22: 424-430.

32. McKenzie FE, Prudhomme WA, Magill AJ, Forney JR, Permpanich B, Lucas C, Gasser RA Jr, Wongsrichanalai C, 2005. White blood cell counts and malaria. J Infect Dis 192: 323-330.

33. McKenzie FE, Bossert WH, 1998. The optimal production of gametocytes by Plasmodium falciparum. J Theor Biol 193: 419-428.

34. Bruce MC, Donnelly CA, Alpers MP, Galinski MR, Barnwell JW, Walliker D, Day KP, 2000. Cross-species interactions between malaria parasites in humans. Science 287: 845.

35. Taylor LH, Walliker D, Read AF, 1997. Mixed-genotype infections of the rodent malaria Plasmodium chabaudi are more infectious to mosquitoes than single-genotype infections. Parasitology 115: 121-132.

36. Butcher G, 1998. Cross-species immunity in malaria. Parasitol Today 14: 166.

37. Smith T, Genton B, Baea K, Gibson N, Narara A, Alpers MP, 2001. Prospective risk of morbidity in relation to malaria in- 
fection in an area of high endemicity of multiple species of Plasmodium. Am J Trop Med Hyg 64: 262-267.

38. Bruce MC, Day KP, 2002. Cross-species regulation of malaria parasitaemia in the human host. Curr Opin Microbiol 5: 431437.

39. Boyd MF, Kitchen SF, 1937. Simultaneous inoculation with Plasmodium vivax and Plasmodium falciparum. Am J Trop Med Hyg 17: 855-861.

40. Collins WE, Skinner JC, Richardson BB, Stanfill PS, 1975. Studies on the transmission of simian malaria. VI. Mosquito infection and sporozoite transmission of Plasmodium fragile. $J$ Parasitol 61: 718-721.

41. Collins WE, Warren M, Skinner JC, Richardson BB, Kearse TS, 1979. Effect of sequential infection with Plasmodium vivax and P. falciparum in the Aotus trivirgatus monkey. J Parasitol 65: 605-608.
42. Schneider P, Bousema JT, Gouagna LC, Otieno S, van de VegteBolmer M, Omar S, Sauerwein R, 2007. Submicroscopic Plasmodium falciparum gametocyte densities frequently result in mosquito infection. Am J Trop Med Hyg 76: 470-474.

43. Terlouw DJ, Courval JM, Kolczak MS, Rosenberg OS, Oloo AJ, Kager PA, Lal AA, Nahlen BL, ter Kuile FO, 2003. Treatment history and treatment dose are important determinants of sulfadoxine-pyrimethamine efficacy in children with uncomplicated malaria in Western Kenya. J Infect Dis 187: 467-476.

44. Snounou G, Pinheiro L, Goncalves A, Fonseca L, Dias F, Brown KN, do Rosario V, 1993. The importance of sensitive detection of malaria parasites in the human and insect hosts in epidemiological studies, as shown by the analysis of field samples from Guinea Bissau. Trans R Soc Trop Med Hyg 87: 649-653. 